\title{
CD166 plays a pro-carcinogenic role in liver cancer cells via inhibition of FOXO proteins through AKT
}

\author{
WENJUN YU $^{1 *}$, JIAYI WANG $^{1 *}$, LIFANG MA $^{1}$, XUN TANG $^{1}$, YONGXIA QIAO $^{3}$, \\ QUIHUI PAN $^{2}$, YONGCHUN YU ${ }^{4}$ and FENYONG SUN ${ }^{1}$
}

\begin{abstract}
Departments of ${ }^{1}$ Clinical Laboratory Medicine and ${ }^{2}$ Central Laboratory, Shanghai Tenth People's Hospital of Tongji University, Shanghai 200072; ${ }^{3}$ School of Public Health, Shanghai Jiaotong University, Shanghai 200025;

${ }^{4}$ Shanghai Municipal Hospital of Traditional Chinese Medicine Affiliated to

Shanghai TCM University, Shanghai 200071, P.R. China
\end{abstract}

Received February 14, 2014; Accepted March 21, 2014

DOI: $10.3892 /$ or.2014.3226

\begin{abstract}
Cluster of differentiation 166 (CD166) is a cell surface membrane protein, which is regarded as a valuable prognostic marker in several types of epithelial tumors. We previously reported that CD166 exerts its pro-carcinogenic role by enhancing YAP function in liver cancer cells. However, YAP cannot completely rescue the increased anti-carcinogenic effects by gene silencing of CD166, whose downstream effectors require further investigation. Here, we found that knockdown of CD166 inhibits phosphorylation of anti-carcinogenic FOXO proteins. Overexpression of CD166 led, not only to a faster protein degradation rate, but also a more accumulated ubiquitination of FOXO compared to the control. Moreover, overexpression of CD166 facilitated FOXO protein localization from the nuclear fraction to the cytosolic fraction, suggesting that CD166 modulates FOXO protein stability through alteration of their subcellular localization. In addition, simultaneous overexpression of CD166 partially reversed the evoked anti-carcinogenic effects by overexpression of FOXO both in vitro and in vivo. Furthermore, CD166 knockdown-induced anti-carcinogenic effects and dephos-
\end{abstract}

Correspondence to: Professor Yongchun Yu, Shanghai Municipal Hospital of Traditional Chinese Medicine Affiliated to Shanghai TCM University, No. 274 Middle Zhijiang Road, Shanghai 200071, P.R. China

E-mail: yueyongchun88@163.com

Dr Fenyong Sun, Department of Clinical Laboratory Medicine, Shanghai Tenth People's Hospital of Tongji University, No. 301 Middle Yanchang Road, Shanghai 200072, P.R. China

E-mail: sunfenyong@126.com

*Contributed equally

Abbreviations: CD166, cluster of differentiation 166; CHX, cycloheximide; FOXO, forkhead box O; shRNA, small hairpin RNA

Key words: CD166, PI3K/AKT, tumorigenesis, protein stability, liver cancer phorylation of FOXO proteins were rescued by overexpression of AKT. In liver cancer tissues, we also observed that higher expression levels of CD166, phospho-AKT, total AKT and phospho-FOXO were correlated with lower expression levels of total FOXO, suggesting that the upregulation of CD166 leads to the activation of AKT, which in turn facilitates phosphorylation and degradation of FOXO. Taken together, our data demonstrate that AKT is an inter-mediator between the upstream regulator, CD166, and downstream effector, FOXO, in liver cancer cells. Disrupting the relationship between CD166 and the AKT/FOXO axis may serve as a novel therapeutic target for liver cancer patients.

\section{Introduction}

Cluster of differentiation 166 (CD166) is a cell surface member of the immunoglobulin superfamily (1), which is overexpressed and regarded as a valuable prognostic marker of disease progression and poor survival in several types of epithelial tumors (2-4). Gene silencing of CD166 decreases the concentration of $\mathrm{Bcl}-2$ and increases the level of apoptosis (PARP, active caspase-7) (5). We previously reported that the activation of anti-apoptotic canonical NF- $\kappa \mathrm{B}$ signaling greatly induces CD166 expression in liver cancer cells after serum deprivation (6), suggesting its important roles in regulating apoptosis. Most recently, we revealed that CD166 can exert its anti-apoptotic role by enhancing YAP function, demonstrating that CD166 is an upstream regulator of YAP (7). However, overexpression of YAP cannot completely rescue the increased anti-carcinogenic effects evoked by knockdown of CD166 (7). Thus, the downstream regulation of the CD166 pro-carcinogenic function needs to be further explored.

The forkhead box transcription factor superfamily consists of 19 subclasses of FOX genes, FOXA-FOXS (8). The FOX transcription factors that belong to the other $(\mathrm{O})$ subfamily (FOXO) include four members (FOXO1, 3, 4, 6) in mammals (8). Overexpression of FOXO proteins inhibits tumor growth in vitro and tumor size in vivo (9). The nuclear accumulation of FOXO proteins was found to suspend cell cycle progression and promote apoptosis in breast cancers $(9,10)$. Recent research efforts also provide new insights that FOXO 
proteins appear to present antitumor properties in liver cancer, including induction of the expression of pro-apoptotic genes, or interfering with signaling cascades commonly altered in this disease such as Wnt/ $\beta$-catenin, PI3K/AKT/mTOR or MAPK pathways (11). However, the upstream regulation of FOXO functions, particularly those involving cell membrane proteins are still largely unknown in liver cancer cells.

In the present study, we found that CD166 exerts its procarcinogenic role via the inhibition of FOXO proteins, i.e. CD166 facilitates phosphorylation, cytosolic accumulation and instability of FOXO proteins. The anti-carcinogenic function of FOXO proteins can be reversed by CD166. Moreover, our data also demonstrate that AKT is an inter-mediator between the upstream regulator, CD166, and downstream effector, FOXO, in liver cancer cells. Disruption of the relationship between CD166 and the AKT/FOXO axis may serve as a novel therapeutic target for liver cancer patients.

\section{Materials and methods}

Cell culture. Bel-7402, SMMC-7721, Chang Liver and HL-7702 cells were cultured in DMEM supplemented with 5\% fetal bovine serum (FBS). Cells were treated by Wortmannin (50 $\mu \mathrm{M}$; Cayman, Ann Arbor, MI, USA), LY294002 [20 $\mu \mathrm{M}$; Cell Signaling Technology (CST), Boston, MA, USA], cycloheximide (CHX, $50 \mu \mathrm{g} / \mathrm{ml}$; Sigma-Aldrich, St. Louis, MO, USA) or MG132 (25 $\mu \mathrm{M}$; Cayman) 1-24 h before harvest.

shRNA and protein expressing plasmids. Lentiviral CD166shRNA1 was purchased from Open Biosystems (Huntsville, AL, USA; catalog no. TRCN0000150706). CD166-shRNA2 and AKT-shRNA were cloned into pLKO.1 lentiviral plasmid using the following primers: CD166-sh2-F, CCGGTCAAG CAACCATCTAAACCTGCTCGAGCAGGTTTAGATGGT TGCTTGATTTTTG and CD166-sh2-R, AATTCAAAAAT CAAGCAACCATCTAAACCTGCTCGAGCAGGTTTAG ATGGTTGCTTGA; AKT-shRNA-F, CCGGAACTCCTCA AGAATGATGGCACTCGAGTGCCATCATTCTTGAGGA GTTTTTTTG and AKT-shRNA-R, AATTCAAAAAAACT CCTCAAGAATGATGGCACTCGAGTGCCATCATTCTT GAGGAGTT. AKT-Myc was cloned into pGIPZ based lentiviral plasmid using the primers as follows: AKT-Myc-F, GGTCGCTAGCAGCGACGTGGCTATTGTGAA and AKT-Myc-R, GTATCCTGCAGGTTACAGATCTTCTTCA GAAATAAGTTTTTGTTCGGCCGTGCCGCTGGCCGAT. The CD166-HA was cloned into pcDNA3.1(+) plasmid using the following primers: CD166-HA-F, GTACGGATCCCACC AAGAAGGAGGAGGAAT and CD166-HA-R, GTACCTCG AGTTAAGCGTAGTCTGGGACGTCGTATGGGTAGGCT TCAGTTTT. The pTEN-HA-expressing plasmid was a gift from Dr Xuqian Fang (Shanghai Jiaotong University, Shanghai, China), and the FOXO1 and FOXO3a protein-expressing plasmids were purchased from OriGene (Beijing, China).

Western blotting (WB). Proteins were resolved on SDS-PAGE gels followed by standard WB. Primary antibodies used were: anti-CD166 (Epitomics, Burlingame, CA, USA; catalog no 3133), anti-HA [Cell Signaling Technology (CST); catalog no. 3724], anti-Myc (CST; catalog no. 2278), antiubiquitin (CST; catalog no. 3936), anti-AKT (Epitomics, catalog no. 2957), anti-p-AKT substrate (CST; catalog no. 9614), anti-GAPDH (CST; catalog no. 5174), anti-p-FOXO3a (S253) (CST; catalog no. 9466), anti-FOXO3a (CST; catalog no. 2497), anti-p-FOXO1 (T24)/p-FOXO3a (T32) (CST; catalog no. 9464), anti-p-FOXO1 (S256) (CST; catalog no. 9461), antiFOXO1 (CST; catalog no. 2880), anti- $\beta$-tublin (Epitomics; catalog no. 1879), anti-p-AKT (CST; catalog no. 2965) and anti-histone H3 (Hua'an Hangzhou, China; catalog no. R1105). For extracting nuclear and cytosolic fractions of cells, a kit from Active Motif (Carlsbad, CA, USA) was used.

Immunofluorescence (IF). For IF, cells were fixed by $4 \%$ paraformaldehyde (PFA) for $15 \mathrm{~min}$, washed with PBS and blocking buffer (3\% FBS $+1 \%$ HISS $+0.1 \%$ Triton X-100) and then incubated overnight at $4^{\circ} \mathrm{C}$ in primary antibodies against HA (CST; catalog no 2367) and FOXO3a (Epitomics; catalog no. 3280). Alexa Fluor 488 or 555 fluorescent-conjugated secondary antibodies (Life Technologies, Carlsbad, CA, USA) were used for detection.

Immunohistochemistry (IHC). Following deparaffinization and rehydration of the tissue sections, antigen retrieval was performed at $100^{\circ} \mathrm{C}$ for $2 \mathrm{~h}$ with Tris-EDTA buffer, pH 6.0 (Beyotime). Endogenous peroxidase was blocked with 3\% peroxide for $20 \mathrm{~min}$, followed by additional rinses in PBS for $3 \times 5 \mathrm{~min}$. Sections were then blocked in a buffer containing $5 \%$ BSA and $0.1 \%$ Triton X-100 and incubated overnight in primary antibodies against CD166 (Epitomics, Burlingame, CA, USA; catalog no. 3133) or FOXO3a (Epitomics; catalog no 3280). Signal detection was accomplished using the Vectastain ABC kit (Vector Laboratories, Burlingame, CA, USA).

Cell proliferation, caspase-3/7 activity and soft agar assays. Cell proliferation was measured by an MTT-based proliferation assay, as previously described (12). Caspase-3/7 activity was determined using the Caspase-Glo 3/7 assay system (Promega, Madison, WI, USA). Anchorage-independent soft agar growth assay and quantitative RT-PCR was performed as previously described (12).

Xenograft mouse model. Bel-7402 cells $\left(5 \times 10^{6}\right)$ expressing proteins as indicated were subcutaneously injected into athymic nude mice (Bikai, Shanghai, China). Tumor size was measured every six days using a caliper, and the tumor volume was calculated as $0.5 \times \mathrm{L} \mathrm{x} \mathrm{W}^{2}$, with $\mathrm{L}$ indicating length and $\mathrm{W}$ indicating width. The mice were euthanized at 24 days after injection.

\section{Results}

CD166 regulates phosphorylation of FOXO proteins. We previously reported that silencing of CD166 can induce apoptosis (7). Notably, activation of FOXO proteins can also induce apoptosis (13). In addition, FOXO protein activity can be inactivated through phosphorylation (8). Thereby, we tested whether CD166 has a role in the regulation of phosphorylation of FOXO proteins. Compared to the control, dephosphorylation of both FOXO1 and FOXO3a was detected in Bel-7402 cells following CD166 knockdown (Fig. 1A). Similar results were also obtained in SMMC-7721 cells (Fig. 1B). Moreover, it 


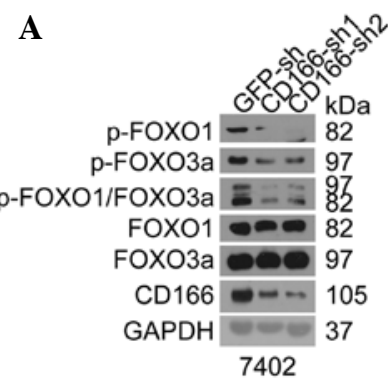

D

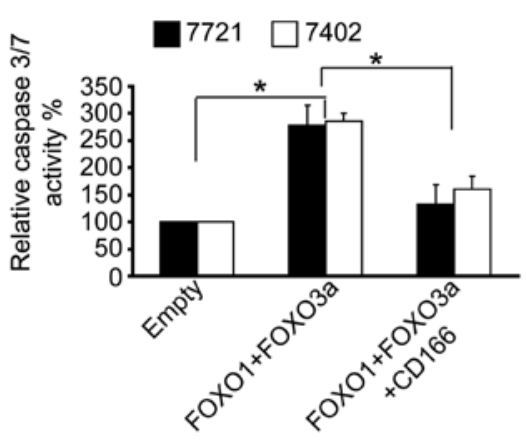

B

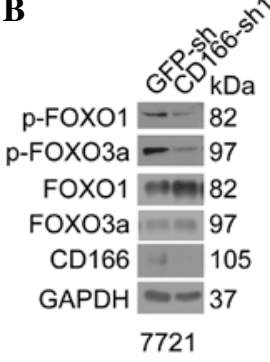

C

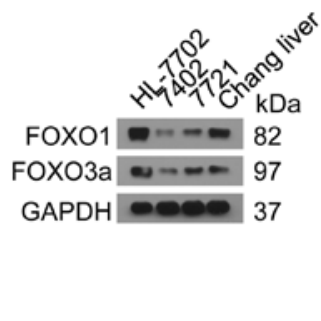

$\mathbf{E}$

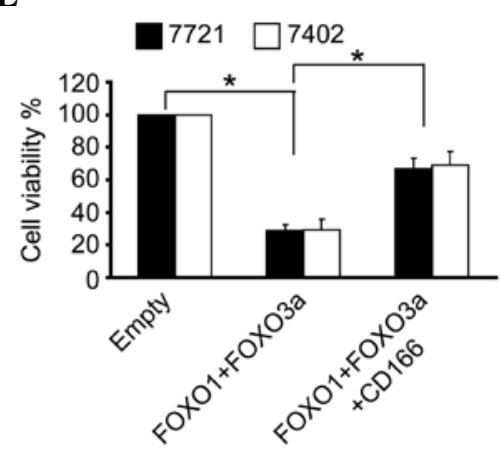

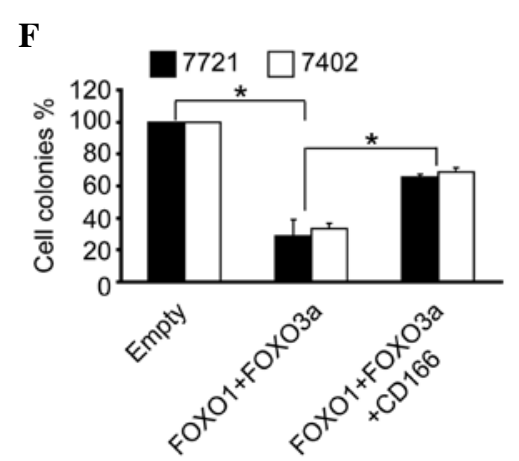

Figure 1. CD166 promotes phosphorylation of FOXO proteins and reverses their anti-carcinogenic property. (A and B) Silencing of CD166 reduced phosphorylation of FOXO proteins. Phosphorylated FOXO and total FOXO proteins as indicated in the control (infected with GFP-shRNA) and Bel-7402 (A) or SMMC-7721 (B) cells with CD166 knockdown (infected with CD166-sh1 or sh2). (C) Expression patterns of FOXO proteins. Western blots of different proteins as indicated in the different cell lines as indicated. (D) Overexpression of FOXO1 and FOXO3a induced apoptosis, which was rescued by overexpression of CD166, as measured by caspase-3/7 activity. (E and F) Relative cell proliferation and transformation activities were measured by MTT (E) and anchorageindependent soft agar colony formation assays (F) in SMMC-7721 and Bel-7402 cells simultaneously expressing FOXO1 and FOXO3a with or without ectopic expression of CD166. Data from panels D-F are shown as mean \pm SD from three independent experiments. The activities of the control (transfected with empty plasmid) were arbitrarily set to $100 \%$. "p $<0.05$, indicates statistic significance analyzed using the Student's t-test. CD166, cluster of differentiation 166; FOXO, forkhead box O; shRNA, small hairpin RNA.

was demonstrated that both FOXO1 and FOXO3a had relative higher levels in normal hepatic cell lines (HL-7702 and Chang Liver) compared to levels in the liver cancer cell lines (Bel7402 and SMMC-7721) (Fig. 1C), suggesting that lower FOXO proteins levels in liver cancer cells may lead to tumorigenesis. Collectively, the above data revealed that CD166 exerts its pro-carcinogenetic role through inhibition of FOXO proteins.

CD166 reverses the anti-carcinogenetic effects induced by FOXO proteins. We examined whether CD166 plays a negative role on FOXO proteins in liver cancer cells. We found that simultaneous overexpression of FOXO1 and FOXO3a decreased cell proliferation compared to the control, as measured by an MTT-based assay and Ki-67 immunostaining (Fig. 1E and data not shown). Furthermore, we found that overexpression of FOXO proteins impaired the ability of these cells to form colonies in soft agar (Fig. 1F), whereas markedly increased apoptosis was noted, as shown by increased caspase3/7 activity and caspase- 3 cleavage by immunostaining (Fig. 1D and data not shown). In addition, we observed that the reduced cell survival and transformative phenotype induced by overexpression of FOXO proteins could be partially rescued by simultaneous ectopic expression of CD166 (Fig. 1D-F). These data indicate that the inhibition of FOXO by CD166 is important for human liver cancer cell growth and survival.

CD166 controls stability and subcellular localization of FOXO proteins. Protein degradation is initialized by target protein modification, such as phosphorylation $(14,15)$. Since CD166 regulated the phosphorylation of FOXO proteins (Fig. 1A and B), we hypothesized that CD166 also modulates degradation of FOXO proteins. It was found that when Bel-7402 cells were treated with the protein synthesis inhibitor, cycloheximide (CHX), the half-life time of FOXO1 and FOXO3a 
A
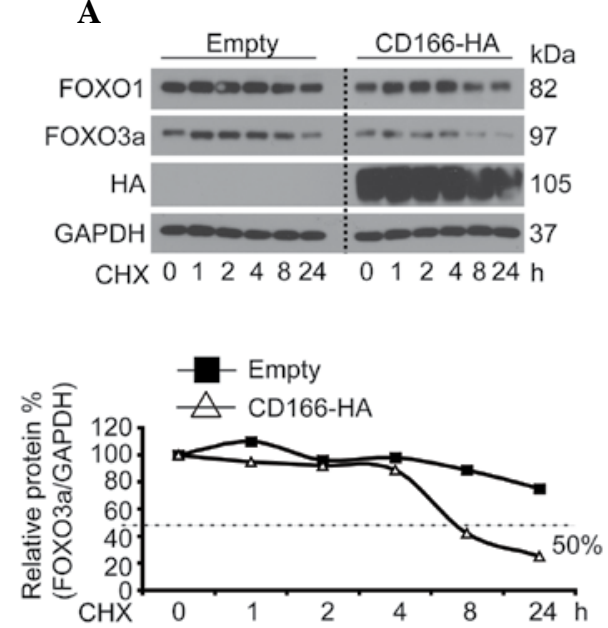

C

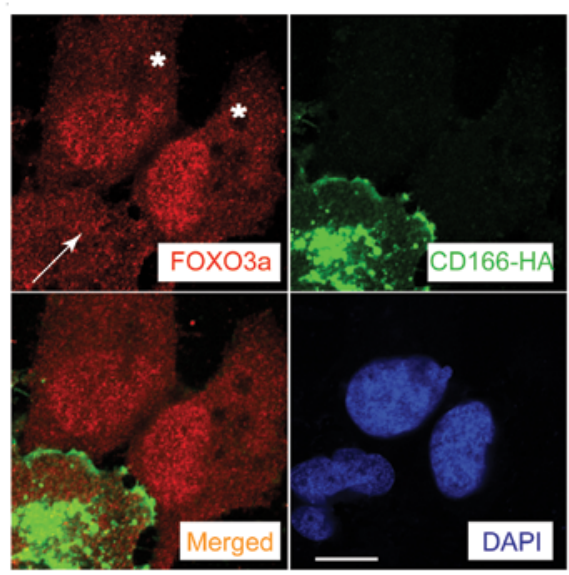

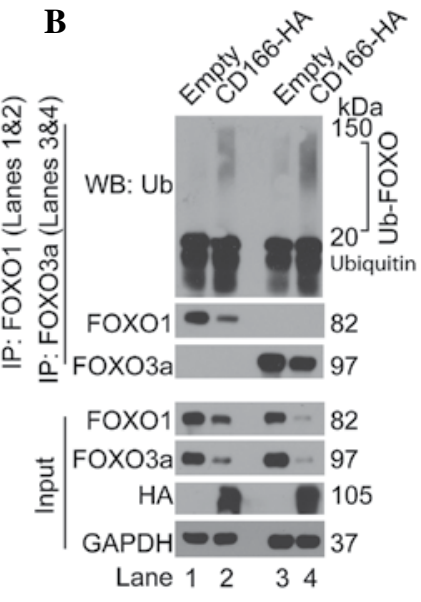

D

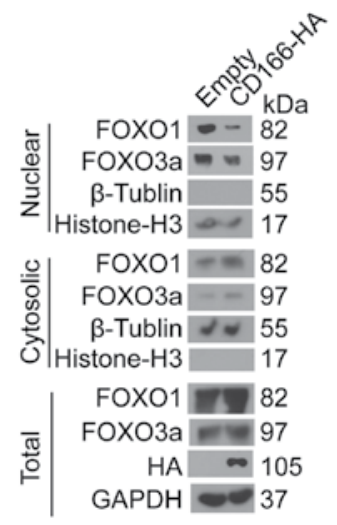

Figure 2. CD166 facilitates degradation and cytosolic localization of FOXO protein. (A) CD166 promotes degradation of FOXO proteins. Protein synthesis was blocked by treatment of CHX (50 $\mu \mathrm{g} / \mathrm{ml})$ for the indicated times in the control (transfected with the empty plasmid) and Bel-7402 cells with CD166 overexpression (transfected with the CD166-HA expression plasmid) and proteins were subjected to western blotting (upper panel). Relative FOXO3a protein levels were quantified by FOXO3a/GAPDH ratio and the $0 \mathrm{~h}$ point was arbitrarily set to $100 \%$ (lower panel). (B) CD166 facilitates ubiquitination of FOXO proteins. Bel-7402 cells transfected with CD166-HA-expressing constructs as indicated for $72 \mathrm{~h}$ were then treated with MG132 (25 $\mu \mathrm{M})$ for $5 \mathrm{~h}$ prior to harvest. Endogenous FOXO proteins were immunoprecipitated by anti-FOXO1 (lanes 1 and 2) and anti-FOXO3a (lanes 3 and 4) antibodies, respectively, and western blotting was performed using anti-Ub and other antibodies as indicated. (C) Overexpression of CD166 reduces nuclear FOXO3a. FOXO3a intracellular localization in Bel-7402 cells with or without CD166-HA overexpression (transfected with the plasmid for $24 \mathrm{~h}$ ) was demonstrated by immunostaining. The arrow indicates cells successfully transfected with the CD166-HA-expressing plasmid, while the asterisks indicate non-transfected cells. Scale bar, $10 \mu \mathrm{m}$. (D) CD166 controls the translocation of FOXO proteins. Subcellular fractions (equal protein) from the control (transfected with the empty plasmid for $24 \mathrm{~h}$ ) and Bel-7402 cells with CD166 overexpression (transfected with the CD166-HA-expressing plasmid for $24 \mathrm{~h}$ ) were subjected to western blotting for proteins as indicated. CD166, cluster of differentiation 166; CHX, cycloheximide; FOXO, forkhead box O.

was $>8$ h. However, FOXO1 and FOXO3a degraded much more rapidly with a half-life time of $\sim 8 \mathrm{~h}$ after overexpression of CD166 (Fig. 2A, upper panel). Compared to FOXO1, the effects of CD166 on FOXO3a were much more obvious (Fig. 2A, lower panel). In addition, in Bel-7402 cells with long exposure ( $72 \mathrm{~h}$ ) to CD166, reduced expression accompanied by a greater accumulation of ubiquitinated FOXO1 and FOXO3a was detected compared to the control (for FOXO1, lane 2 vs. 1 and for FOXO3a, lane 4 vs. 3) (Fig. 2B), suggesting that CD166 is a regulator of ubiquitintation and degradation of FOXO proteins. As known, inactivation of FOXO proteins leads to their accumulation in the cytoplasm (8). To ascertain whether CD166 affects the subcellular localization of FOXO, Bel-7402 cells were transfected with CD166-HA-expressing plasmids. It was detected that nuclear FOXO3a expression was significantly reduced in the Bel-7402 cells with CD166-HA overexpression compared to the control (Fig. 2C). This obser- vation was confirmed by fractionation studies, which revealed that overexpression of CD166 facilitated FOXO protein localization from the nuclear fraction to the cytosolic fraction (Fig. 2D). Taken together, the data demonstrate that CD166 modulates FOXO protein stability through alteration of their subcellular localization.

CD166 promotes liver cancer cell growth through inhibition of FOXO in vivo. On the basis of the evidence that CD166 reduces protein stability and expression (Fig. 2), we investigated the growth of Bel-7402 clones after injection into athymic mice. Compared to the control (transfected with the empty plasmid), Bel-7402 cells with CD166 overexpression exhibited a relatively higher tumor growth rate (Fig. 3A). In comparison, Bel-7402 cells with FOXO (FOXO1 and FOXO3a) overexpression effectively prevented tumor growth, yet this effect was rescued by simultaneous overexpression of CD166 (Fig. 3A), 

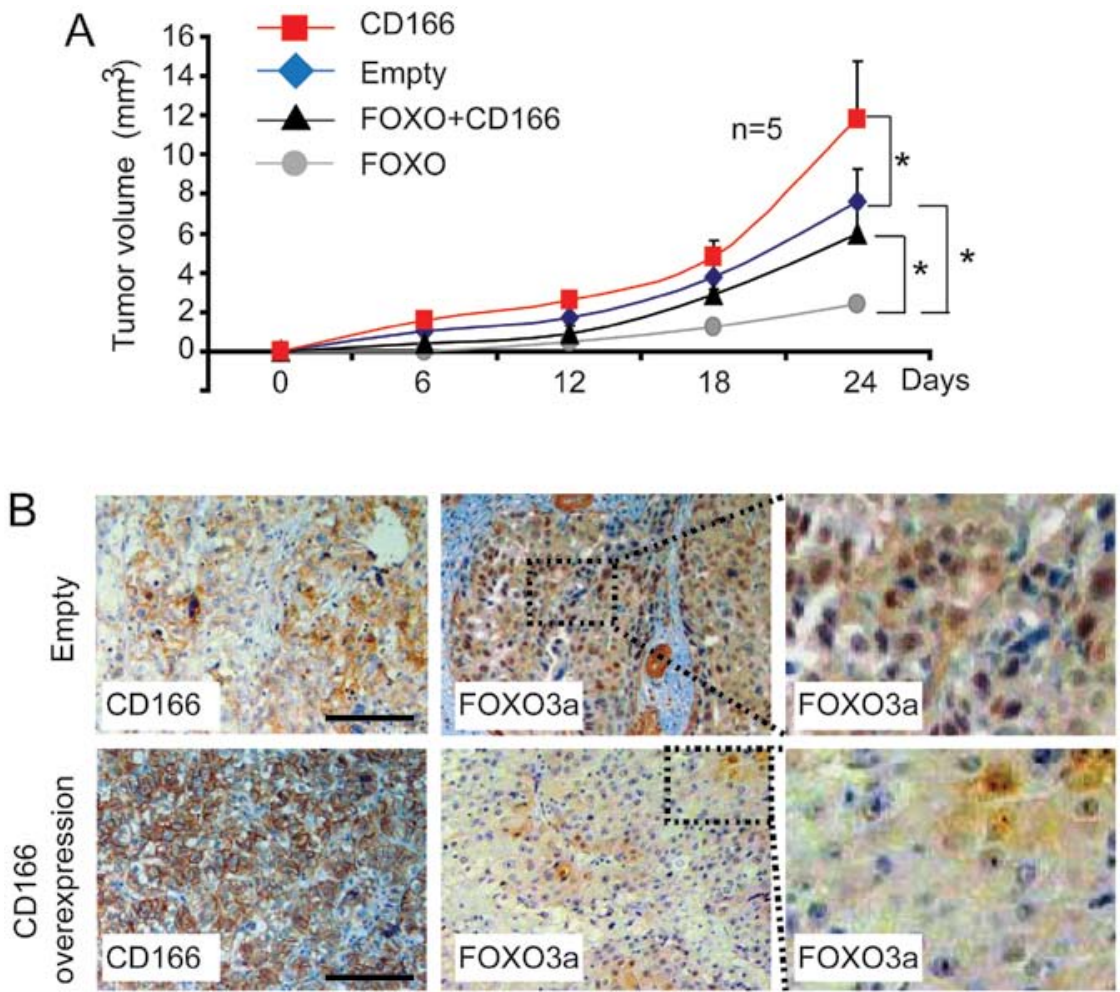

Figure 3. CD166 reduces nuclear FOXO protein expression and rescues their anti-carcinogenic property in xenograft models. (A) Ectopic expression of CD166 rescues FOXO protein-inhibited Bel-7402 cell growth in xenograft models. Bel-7402 cells $\left(5 \times 10^{6}\right)$ expressing different protein as indicated were initially subcutaneously injected into athymic nude mice. Tumor volumes were measured for 24 days after subcutaneous injection ( $\mathrm{n}=5$ per group). FOXO indicates simultaneously overexpression of FOXO1 and FOXO3a. " $\mathrm{p}<0.05$, indicates statistical significance analyzed using the Student's t-test. (B) Representative IHC images of control (transfected with empty plasmids) and xenograft tissues with CD166 ectopical expression from panel (A) stained using anti-CD166 and anti-FOXO3a antibodies. Scale bar, $200 \mu \mathrm{m}$. CD166, cluster of differentiation 166; FOXO, forkhead box O.

thereby confirming the close relationship between FOXO and CD166 in vivo. To ascertain whether CD166 contributes to the inhibition of FOXO in vivo, we stained sections from the xenografts using anti-FOXO3a antibodies. Similar to the data shown in Fig. 2B and C, the protein expression of FOXO3a, particularly the nuclear fraction of FOXO3a was significantly downregulated in xenograft tissue with CD166 overexpression compared to the control (transfected with the empty plasmids) (Fig. 3B), suggesting that long-term exposure to the overexpression of CD166 leads to translocation from the nucleus to the cytoplasm and instability of FOXO proteins.

AKT regulates FOXO proteins in liver cancer cells. Emerging evidence suggests that AKT controls the activity of FOXO proteins $(8,16,17)$. However, to the best of our knowledge, there is no direct evidence to support the conclusion that AKT regulates FOXO proteins in liver cancer cells. Thus, we investigated the effect of AKT on FOXO in Bel-7402 cells. Compare to the control, we found that phosphorylation of FOXO1 and FOXO3a was greatly induced by overexpression of AKT (Fig. 4A), whereas it was reduced after knockdown of AKT (Fig. 4B). By using chemical PI3K/AKT inhibitors, LY294002 and Wortmannin, respectively, we found that phosphorylation of both FOXO1 and FOXO3a was markedly reduced (Fig. 4C). Furthermore, when endogenous PI3K/AKT inhibitor, pTEN, was overexpressed, phosphorylation of FOXO1 and FOXO3a was also inhibited in a dose-dependent manner (Fig. 4D), suggesting that FOXO proteins are also regulated by AKT in liver cancer cells. As shown in Fig. 4E, we confirmed that AKT activity could be downregulated after knockdown of CD166, as the phosphorylation of AKT substrates was markedly reduced in the Bel-7402 cells with CD166 knockdown compared to the control suggesting that the regulation of FOXO by CD166 may be AKT dependent.

CD166 regulates FOXO proteins via AKT. We then investigated whether the phosphorylation of FOXO proteins controlled by CD166 is AKT dependent. It was found that knockdown of CD166-induced dephosphorylation of FOXO proteins could be rescued by simultaneous overexpression of AKT (Fig. 5A). Moreover, the induced caspase-3/7 activity, reduced cell proliferation and soft agar colony formation by depletion of CD166 was partially reversed after overexpression of AKT (Fig. 5B-D). Thus, we proposed that AKT may act as an inter-mediator between the upstream regulator, CD166, and the downstream effector, FOXO. Next, we tested the protein expression patterns of FOXO, AKT and CD166 in liver cancers and their adjacent normal liver tissues. Compared to the adjacent normal liver tissues, higher expression levels of CD166, p-AKT, total AKT and p-FOXO1/3a were correlated with lower expression levels of total FOXO1/3a in liver cancer tissues (Fig. 5E), suggesting that upregulation of CD166 leads to induction of AKT, which in turn facilitates phosphorylation and degradation of FOXO in liver cancer. 

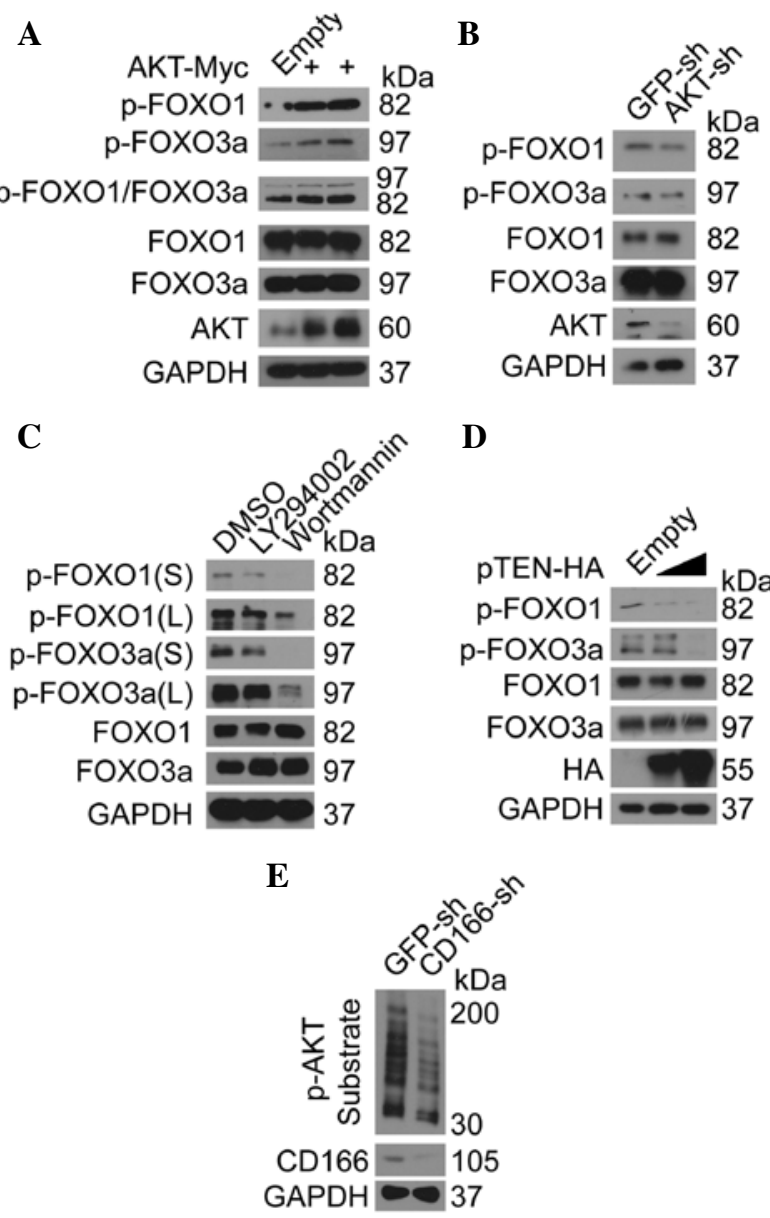

Figure 4. Phosphorylation of FOXO proteins by AKT in liver cancer cells. (A) Overexpression of AKT induces phosphorylation of FOXO proteins. Western blotting of proteins as indicated in the control (transfected with empty plasmid) and Bel-7402 cells with AKT-Myc overexpression (infected with AKT-Myc for $48 \mathrm{~h}$ ). (B) Knockdown of AKT reduces phosphorylation of FOXO proteins. Western blotting of proteins as indicated in control (infected with GFP-shRNA) and Bel-7402 cells with AKT knockdown (infected with AKT-shRNA). (C) Chemical PI3K/AKT inhibitors reduce phosphorylation of FOXO proteins. Bel-7402 cells were treated with either DMSO, Wortmannin $(50 \mu \mathrm{M})$, or LY294002 $(20 \mu \mathrm{M}) 24 \mathrm{~h}$ prior to harvest. Cell lysates were then subjected to western blotting for detection of different proteins as indicated. S, shorter exposure and L, longer exposure. (D) Overexpression of pTEN reduces the phosphorylation of FOXO proteins. Western blotting of proteins as indicated in the control (transfected with empty plasmid for $24 \mathrm{~h}$ ) and Bel-7402 cells with pTEN-HA overexpression (transfected with pTEN-HA-expressing plasmid for $24 \mathrm{~h}$ ). (E) Knockdown of CD166 reduces AKT activity. Phosphorylation of AKT substrates was detected in the control (infected with GFP-shRNA) and Bel-7402 cells with CD166 knockdown (infected with CD166-sh1) by western blotting using anti-p-AKT substrate antibodies. CD166, cluster of differentiation 166; FOXO, forkhead box O; shRNA, small hairpin RNA.

\section{Discussion}

In the present study, we describe a close relationship between CD166 and FOXO proteins (Fig. 5F). These two types of proteins play opposing roles in the regulation of tumorigenesis in liver cancer $(6,7,11,18,19)$. Nuclear localization of FOXO proteins induces expression of anti-carcinogenic genes (11), whereas CD166 facilitates translocation of FOXO proteins from the nucleus to the cytoplasm (Fig. 2C and D). CD166 maintains anti-apoptotic $\mathrm{Bcl}-2$ protein expression, suggesting that the anti-apoptotic function of CD166 is partially dependent
$\mathbf{A}$
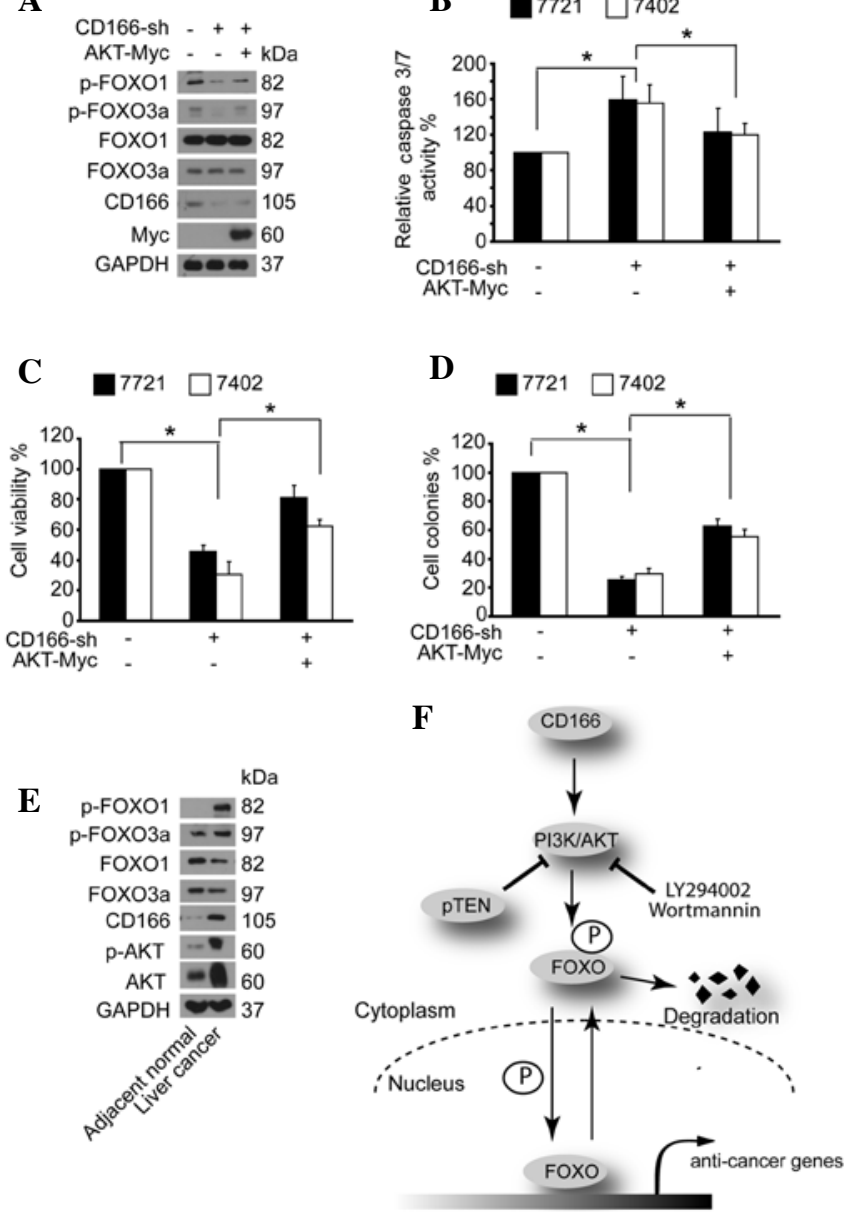

Figure 5. Regulation of FOXO proteins by CD166 is via AKT. (A) AKT rescues CD166 knockdown-induced dephosphorylation of FOXO proteins. Western blotting of indicated proteins in Bel-7402 cells following different treatments as indicated. (B) AKT rescues induced apoptosis by knockdown of CD166. Caspase-3/7 activities in the control and Bel-7402 or SMMC-7721 cells infected with lentivirus as indicated were measured using Caspase-Glo 3/7 assay system. (C and D) Relative cell proliferation and transformation activities were measured by MTT (C) and anchorage-independent soft agar colony formation assays (D) in control and SMMC-7721 or Bel-7402 cells infected with lentivirus as indicated. Panels B-D, the data are shown as mean \pm SD from three independent experiments. The activities of the control (infected with empty lentivirus) were arbitrarily set to $100 \%$. ${ }^{*} \mathrm{p}<0.05$, indicates statistic significance analyzed using the Student's t-test. (E) Expression profiling of proteins. Western blotting of proteins as indicated in a mix pool of liver cancer (equal amount) and corresponding adjacent normal tissues (equal amount) from 10 patients. (F) Possible mechanism underlying how CD166 exerts its role in the regulation of FOXO proteins. CD166, cluster of differentiation 166; FOXO, forkhead box O.

on Bcl-2 (7). Notably, overexpression of Bcl-2 also diminishes death induced by expression of FOXO proteins (20). We previously reported that the anti-apoptotic function of CD166 is via enhancement of both expression and activity of oncoprotein, YAP (7). The degradation of YAP can be protected by TRIB2 (21-22), a protein that is also capable of inhibiting and reducing nuclear FOXO proteins (23). Thus, we consider that CD166 plays a similar role to that of TRIB2. YAP is a pro-carcinogenic protein $(7,12)$, while FOXO proteins are anti-carcinogenic in liver cancer. However, whether and how YAP antagonizes FOXO still remains unknown and needs further exploration. 
It has been reported that AKT phosphorylates FOXO3a at $\mathrm{T} 32$ and $\mathrm{S} 253$ and FOXO1 at $\mathrm{T} 24$ and S256, respectively, which are conserved from Caenorhabditis elegans to mammals (24). Notably, these phosphorylation sites can also be regulated by CD166 (Fig. 1A and B). Furthermore, the anticarcinogenic effects and the regulation of phosphorylation of FOXO proteins by knockdown of CD166 can be reversed by AKT (Fig. 5A-D), providing another evidence that CD166 can regulate AKT.

In summary, our data indicate that the CD166/AKT axis modulates tumorigenesis via promotion of phosphorylation and facilitation of degradation, ubiquitination and cytosolic accumulation of FOXO proteins in liver cancer cells. Further exploration of the interplay among these important signaling pathways may lead to more effective therapeutic strategies for liver cancer.

\section{Acknowledgements}

This study was supported by China National 973 Projects (grant nos. 20111812 and 20110402), the Natural Science Foundation of China (grant nos. 81272292 and 81301689) and Climbing Training Program (to J.W.) from Shanghai Tenth People's Hospital.

\section{References}

1. van Kempen LC, Nelissen JM, Degen WG, Torensma R Weidle UH, Bloemers HP, Figdor CG and Swart GW: Molecular basis for the homophilic activated leukocyte cell adhesion molecule (ALCAM)-ALCAM interaction. J Biol Chem 276: 25783-25790, 2001.

2. Weichert W, Knösel T, Bellach J, Dietel M and Kristiansen G: ALCAM/CD166 is overexpressed in colorectal carcinoma and correlates with shortened patient survival. J Clin Pathol 57: 1160-1164, 2004.

3. Burkhardt M, Mayordomo E, Winzer KJ, Fritzsche F, Gansukh T, Pahl S, Weichert W, Denkert C, Guski H and Dietel MG: Cytoplasmic overexpression of ALCAM is prognostic of disease progression in breast cancer. J Clin Pathol 59: 403-409, 2006.

4. Kahlert C, Weber H, Mogler C, Bergmann F, Schirmacher P, Kenngott HG, Matterne U, Mollberg N, Rahbari NN, Hinz U, Koch M, Aigner $M$ and Weitz $J$ : Increased expression of ALCAM/CD166 in pancreatic cancer is an independent prognostic marker for poor survival and early tumour relapse. $\mathrm{Br} \mathrm{J}$ Cancer 101: 457-464, 2009.

5. Jezierska A, Matysiak W and Motyl T: ALCAM/CD166 protects breast cancer cells against apoptosis and autophagy. Med Sci Monit 12: BR263-BR273, 2006.

6. Wang J, Gu Z, Ni P, Qiao Y, Chen C, Liu X, Lin J, Chen N and Fan Q: NF- $\mathrm{B}$ P50/P65 hetero-dimer mediates differential regulation of CD166/ALCAM expression via interaction with micoRNA-9 after serum deprivation, providing evidence for a novel negative auto-regulatory loop. Nucleic Acids Res 39: 6440-6455, 2011.

7. Ma L, Wang J, Lin J, Pan Q, Yu Y and Sun F: Cluster of differentiation 166 (CD166) regulated by phosphatidylinositide 3-kinases $(\mathrm{PI} 3 \mathrm{~K}) / \mathrm{AKT}$ signaling to exert its anti-apoptotic role via yes associated protein (YAP) in liver cancer. J Biol Chem: Jan 30, 2014 (Epub ahead of print). doi: 10.1074/jbc.M113.524819.
8. Zhang $\mathrm{X}$, Tang $\mathrm{N}$, Hadden TJ and Rishi AK: Akt, FoxO and regulation of apoptosis. Biochim Biophys Acta 1813: 1978-1986, 2011.

9. Medema RH, Kops GJ, Bos JL and Burgering BM: AFX-like Forkhead transcription factors mediate cell-cycle regulation by Ras and PKB through p27 ${ }^{\mathrm{kip} 1}$. Nature 404: 782-787, 2000.

10. Urbich C, Knau A, Fichtlscherer S, Walter DH, Brühl T, Potente M, Hofmann WK, de Vos S, Zeiher AM and Dimmeler S: FOXO-dependent expression of the proapoptotic protein Bim: pivotal role for apoptosis signaling in endothelial progenitor cells. FASEB J 19: 974-976, 2005.

11. Carbajo-Pescador S, Mauriz JL, García-Palomo A and González-Gallego J: FoxO proteins: regulation and molecular targets in liver cancer. Curr Med Chem 21: 1231-1246, 2014.

12. Wang J, Ma L, Weng W, Qiao Y, Zhang Y, He J, Wang H, Xiao W, Li L, Chu Q, Pan Q, Yu Y and Sun F: Mutual interaction between YAP and CREB promotes tumorigenesis in liver cancer. Hepatology 58: 1011-1020, 2013.

13. You H, Yamamoto K and Mak TW: Regulation of transactivation-independent proapoptotic activity of p53 by FOXO3a. Proc Natl Acad Sci USA 103: 9051-9056, 2006.

14. Yamashita M, Ying SX, Zhang GM, Li C, Cheng SY, Deng CX and Zhang YE: Ubiquitin ligase Smurf1 controls osteoblast activity and bone homeostasis by targeting MEKK2 for degradation. Cell 121: 101-113, 2005.

15. Sapkota G, Alarcon C, Spagnoli FM, Brivanlou AH and Massague J: Balancing BMP signaling through integrated inputs into the Smad1 linker. Mol Cell 25: 441-454, 2007.

16. Fu $\mathrm{Z}$ and Tindall DJ: FOXOs, cancer and regulation of apoptosis. Oncogene 27: 2312-2319, 2008

17. Hales EC, Taub JW and Matherly LH: New insights into Notch1 regulation of the $\mathrm{PI} 3 \mathrm{~K}-\mathrm{AKT}$-mTOR1 signaling axis: targeted therapy of $\gamma$-secretase inhibitor resistant T-cell acute lymphoblastic leukemia. Cell Signal 26: 149-161, 2014.

18. Lin A, Yao J, Zhuang L, Wang D, Han J, Lam EW, TCGA Research Network and Gan B: The FoxO-BNIP3 axis exerts a unique regulation of $\mathrm{mTORC} 1$ and cell survival under energy stress. Oncogene: Jul 15, 2013 (Epub ahead of print). doi: 10.1038/onc.2013.273.

19. Tao GZ, Lehwald N, Jang KY, Baek J, Xu B, Omary MB and Sylvester KG: Wnt/ $\beta$-catenin signaling protects mouse liver against oxidative stress-induced apoptosis through the inhibition of forkhead transcription factor FoxO3. J Biol Chem 28: 17214-17224, 2013

20. Yusuf I, Zhu X, Kharas MGs, Chen J and Fruman DA: Optimal B-cell proliferation requires phosphoinositide 3-kinase-dependent inactivation of FOXO transcription factors. Blood 104: 784-787, 2004.

21. Wang J, Zhang Y, Weng W, Qiao Y, Ma L, Xiao W, Yu Y, Pan Q and Sun F: Impaired phosphorylation and ubiquitination by p70 S6 kinase (p70S6K) and smad ubiquitination regulatory factor 1 (Smurf1) promote tribbles homolog 2 (TRIB2) stability and carcinogenic property in liver cancer. J Biol Chem 288: 33667-33681, 2013.

22. Wang J, Park JS, Wei Y, Rajurkar M, Cotton JL, Fan Q, Lewis BC, Ji $\mathrm{H}$ and Mao J: TRIB2 acts downstream of Wnt/TCF in liver cancer cells to regulate YAP and $\mathrm{C} / \mathrm{EBP} \alpha$ function. Mol Cell 51: 211-225, 2013.

23. Zanella F, Renner O, García B, Callejas S, Dopazo A, Peregrina S, Carnero A and Link W: Human TRIB2 is a repressor of FOXO that contributes to the malignant phenotype of melanoma cells. Oncogene 29: 2973-2982, 2010.

24. Manning BD and Cantley LC: AKT/PKB signaling: navigating downstream. Cell 129: 1261-1274, 2007. 